\title{
THE CHARACTERISTIC POLYNOMIAL AND DETERMINANT ARE NOT AD HOC CONSTRUCTIONS
}

\author{
R. SKIP GARIBALDI
}

Most people are first introduced to the characteristic polynomial and determinant of a matrix in a linear algebra course as undergraduates. The definition of the characteristic polynomial seen there appears totally ad hoc to my eye. The determinant is usually defined as an alternating sum of products of entries of the matrix (as in Jacobi [Jac41, §4], [Web61, §23], and [Bre01, §6.1]); as the unique map $M_{n}(F) \rightarrow F$ which is multilinear and alternating in the columns and which is 1 on the identity matrix (as in Weierstrass Weil and the books by Hungerford Hun80; Lang; and Dummitt and Foote); or - what amounts to the same - in terms of the representation on the $n$th exterior power $\wedge^{n} F^{n}$ (as in Bou48, Ch. III, $\S 6])$.

Now, the determinant of a linear transformation on $\mathbb{R}^{n}$ is a natural enough object: its absolute value gives the factor by which the transformation enlarges volumes, and its sign says whether or not the map preserves orientation. These properties imply Weierstrass' axioms, cf. Han96 or Lax97, §5]. This is a nice development, but it does not explain the characteristic polynomial. From a more algebraic perspective, it also fails to explain the analogues of the characteristic polynomial and determinant which are known for Hamilton's quaternions and the octonions, as well as why one should use the same formula to define the determinant of matrices over fields other than $\mathbb{R}$.

As we show below, every finite-dimensional algebra $A$ over a field $F$ has a characteristic polynomial and a determinant map, which coincide with the usual ones in the case $A=M_{n}(F)$. The philosophy is the following: a "generic" $n$-by- $n$ matrix has $n$ distinct eigenvalues. (More precisely, one knows a fortiori that amongst the $n$-by- $n$ matrices, those with distinct eigenvalues form a dense open subset. Over $\mathbb{R}$ or $\mathbb{C}$ this is true in the usual topology; it is also true over any infinite field in the Zariski topology [Jac75, §I.9].) For such a matrix, the traditional characteristic polynomial is just the minimal polynomial. The technical device of specialization can be used to extend this notion of characteristic polynomial for a generic matrix to all matrices. This method of defining the characteristic polynomial works for all finite-dimensional $F$-algebras, and the determinant is (up to sign) the constant term of the characteristic polynomial.

The ideas here are not really new; they go back at least to an 1889 paper by Study Stu89]. (See also [Sch91, p. 301].) However, all treatments that I have found do not develop the properties of the general characteristic polynomial (as in Dic38 and Deu48), or they make use of known properties of the characteristic polynomial and determinant for matrices in studying the general characteristic polynomial (as in Alb61, §I.13], Jac68, Jac63, and [Jac43, §5.18]). We use only elementary properties of matrices from the very nice paper Ax195.

Date: 22 March 2002. 
Readers with an algebraic background may argue that one can obtain the characteristic polynomial of an $n$-by- $n$ matrix of an arbitrary field $F$ by applying the structure theory for finitely generated torsion modules over a PID (as is done in Her75, §6.7]). But once one is using that much algebra, the contents of this paper are not so far away, and the results here are much stronger.

\section{The ChARACTERISTIC POLYNOMial}

Definition 1.1. An $F$-algebra is a ring $A$ with identity which is an $F$-vector space such that $\alpha(a b)=(\alpha a) b=a(\alpha b)$ for all $\alpha \in F$ and $a, b \in A$. (Alternately, $A$ is a ring with identity such that there is a monomorphism $F \rightarrow Z(A)$ which maps the identity in $F$ to the identity in $A$.) All algebras that we consider will be finitedimensional as vector spaces over $F$.

Principal examples of $F$-algebras are the $n$-by- $n$ matrices $M_{n}(F)$ and Hamilton's quaternions, which form an $\mathbb{R}$-algebra. There is no requirement that $F$ is the center of $A$; for example, $A$ may be taken to be a finite field extension of $F$. (The following results may also be extended to the much wider class of strictly power-associative $F$-algebras, see [Jac68. This class includes, for example, the octonions and Jordan algebras.)

The only tool we need that is not always discussed in a first year graduate algebra course is the tensor product $\otimes$, for which we refer the reader to any good graduate algebra text. Heuristically, it allows one to make precise the notion of enlarging our base field $F$ : if $K$ is any extension field of $F$, then $M_{n}(F) \otimes_{F} K$ is isomorphic to $M_{n}(K)$ as $K$-algebras.

Let $a_{1}, \ldots, a_{m}$ be an $F$-basis for $A$. Let $R=F\left[t_{1}, \ldots, t_{m}\right]$ for $t_{1}, \ldots, t_{m}$ indeterminates, and let $K$ be the quotient field of $R$. We call $\gamma=\sum_{i} t_{i} a_{i}$ in $A \otimes_{F} K$ a generic element. The $K$-span of $1, \gamma, \gamma^{2}, \ldots$ is a subspace of $A \otimes K$, so it must be finite-dimensional over $K$. Hence there is a nonzero monic polynomial min. poly. $\gamma / K$ in $K[x]$ of smallest degree such that min. poly ${ }_{\gamma} / K(\gamma)=0$, called the minimal polynomial for $\gamma$ over $K$. The usual argument shows that it is unique.

Lemma 1.2. The minimal polynomial min. poly ${ }_{\gamma / K}$ is in $R[x]$, not just $K[x]$.

Proof. Consider the submodules $A_{j}$ of $A \otimes R$ generated by $\left\{1, \gamma, \gamma^{2}, \ldots, \gamma^{j}\right\}$. They form an ascending chain $A_{1} \subseteq A_{2} \subseteq \cdots$. Since $R$ is noetherian (Hilbert's Basis Theorem) and $A \otimes R$ is a finitely-generated $R$-module, this chain must stabilize. That is, $\gamma_{j+1}$ is in $A_{j}$ for some $j$, so $\gamma$ satisfies a monic polynomial $f$ in $R[x]$. 1] Since min. poly $\cdot_{\gamma / K}$ divides $f$ and both are monic, min. poly ${ }_{\gamma / K}$ lies in $R[x]$ by Gauss' Lemma.

Definition 1.3. Write $a \in A$ with respect to the basis $a_{1}, \ldots, a_{m}$ above as $a=$ $\sum_{i} \alpha_{i} a_{i}$. The specialization $t_{i} \mapsto \alpha_{i}$ defines a map $R[x] \rightarrow F[x]$. We call the image of min. poly $\cdot_{\gamma / K}$ in $F[x]$ the characteristic polynomial of a and denote it by ch. poly $a, A / F$ or simply ch. poly $\cdot a$.

\footnotetext{
${ }^{1}$ A more direct argument would be: $R[\gamma]$ is an $R$-submodule of $A \otimes R$, which is finitely-generated as an $R$-module. Hence $\gamma$ is integral over $R$ Hun80, Thm. VIII.5.3]. Unfortunately, the typical proof of this implication invokes determinants, so we use instead that $R$ is noetherian.
} 
Example 1.4 (Upper triangular matrices). Let $A$ be the algebra of $n$-by- $n$ upper triangular matrices over $F$. Write $E_{i j}$ for the matrix whose only nonzero entry is in the $(i, j)$-position. Fix a basis $a_{1}, \ldots, a_{m}$ for $A$ over $F$ whose first $n$ elements are the matrices $E_{i i}$ for $1 \leq i \leq n$, and let $\gamma$ be the generic element constructed above.

Let $I_{n}$ denote the $n$-by- $n$ identity matrix. For each $1 \leq i \leq n$, the matrix $\gamma-t_{i} I_{n}$ has $n-1$ pivot columns in its row-reduced form, hence it has a nonzero kernel. That is, $\gamma$ has an eigenvector in $K^{n}$ with eigenvalue $t_{i}$. Since the $t_{i}$ are distinct elements of $K$, these eigenvectors form a basis for $K^{n}$ and $\gamma$ is similar in $M_{n}(K)$ to the diagonal matrix with diagonal entries $t_{1}, t_{2}, \ldots, t_{n}$. The minimal polynomial of $\gamma$ is $\prod_{i=1}^{n}\left(x-t_{i}\right)$, since similar matrices have the same minimal polynomials. 2]

By specialization, an upper triangular matrix $a \in A$ has characteristic polyno$\operatorname{mial} \prod_{i=1}^{n}\left(x-a_{i i}\right)$.

Proposition 1.5. The characteristic polynomial ch. poly $\cdot_{a, A / F}$ depends only on a, $A$, and $F$ (and not on the choice of basis for $A$ ).

Proof. Suppose that we have another $F$-basis $b_{1}, \ldots, b_{m}$ of $A$ with a corresponding generic element $\varepsilon=\sum_{i} t_{i} b_{i}$. We may write $b_{i}=\sum_{j} g_{i j} a_{j}$ for $g$ an invertible $m$-by- $m$ matrix. Let $f: R \rightarrow R$ be the $F$-automorphism defined by

$$
f\left(t_{j}\right)=\sum_{i} t_{i} g_{i j}
$$

Write $a \in A$ in terms of both bases as

$$
a=\sum_{j} \alpha_{j} a_{j}=\sum_{i} \beta_{i} b_{i} .
$$

We have a diagram

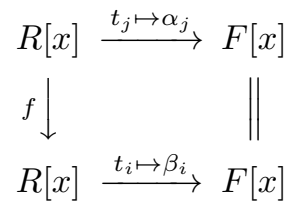

with horizontal arrows the specialization maps. Equation (1.6) gives that $\alpha_{j}=$ $\sum_{i} \beta_{i} g_{i j}$ for all $j$, hence the diagram commutes.

If we begin with min. poly ${ }_{\gamma / K}$ in the upper left, specialization gives ch. poly. ${ }_{a}$ computed with respect to the basis $a_{1}, \ldots, a_{m}$ in $F[x]$. On the other hand, $f$ extends in an obvious way to an automorphism of $A \otimes R$ such that

$$
f(\gamma)=\sum_{j} f\left(t_{j}\right) a_{j}=\sum_{j}\left(\sum_{i} t_{i} g_{i j}\right) a_{j}=\sum_{i} t_{i}\left(\sum_{j} g_{i j} a_{j}\right)=\varepsilon .
$$

Hence $f\left(\right.$ min. poly $\left.\cdot_{\gamma / K}\right)=$ min. poly ${ }_{\varepsilon / K}$. The image of this in $F[x]$ is ch. poly ${ }_{a}$ computed with respect to the basis $b_{1}, \ldots, b_{n}$. The commutativity of the diagram gives the claim.

\footnotetext{
${ }^{2}$ This argument may appear to be excessively long. It is included here to illustrate that we are not making use of determinants.
} 
Lemma 1.7. Let $E$ be a field containing $F$. Then for every $a \in A$, we have

$$
\min \text {. poly } \cdot a / F=\min . \text { poly } \cdot(a \otimes 1) / E
$$

and

$$
\text { ch. poly }{ }_{a, A / F}=\text { ch. poly } \cdot(a \otimes 1),(A \otimes E) / E \cdot
$$

There is a natural map $A \rightarrow A \otimes E$, and $a \otimes 1$ denotes the image of $a$. (The first displayed equation gives that the natural map $A \rightarrow A \otimes E$ is an injection, so in the future we will identify $a$ with $a \otimes 1$.)

Proof. Since $F$ is a field, $a$ generates a free submodule $B$ of $A$ with basis $1, a, a^{2}$, $\ldots, a^{d-1}$ for $d$ the degree of min. poly $\cdot_{a / F}$. Then $B \otimes E$ is a free $E$-module with the same basis, hence the degree of min. poly ${ }_{(a \otimes 1) / E}$ is $\geq d$. Since this polynomial divides min. poly ${ }_{a / F}$, they are the same.

The $F$-basis $a_{1}, \ldots, a_{m}$ of $A$ gives an $E$-basis $a_{1} \otimes 1, \ldots, a_{m} \otimes 1$ of $A \otimes E$, and the generic element constructed from this $E$-basis is $\gamma \otimes 1 \in K \otimes E$. Since the minimal polynomials of $\gamma / K$ and $(\gamma \otimes 1) /(K \otimes E)$ are the same by the preceding paragraph, we get

$$
\text { ch. poly } \cdot a, A / F=\text { ch. poly } \cdot(a \otimes 1),(A \otimes E) / E
$$

by specialization.

In general, we write

$$
\begin{aligned}
\text { ch. poly } \cdot a & (x)=x^{n}-c_{1}(a) x^{n-1}+\cdots \\
& \\
& \cdots+(-1)^{n-1} c_{n-1}(a) x+(-1)^{n} c_{n}(a) .
\end{aligned}
$$

The elements $c_{1}(a)$ and $c_{n}(a)$ play the roles of the trace and determinant of $a$.

Proposition 1.9. Let $A$ be a finite-dimensional F-algebra. Then:

1. (Cayley-Hamilton) ch. poly. ${ }_{a}(a)=0$ for all $a \in A$.

2. If $f: A \rightarrow A$ is a ring automorphism or anti-automorphism of $A$ which restricts to be an automorphism of $F$, then $f\left(c_{i}(a)\right)=c_{i}(f(a))$ for all $a \in A$.

3. $c_{i}(\alpha a)=\alpha^{i} c_{i}(a)$ for $\alpha \in F$ and $a \in A$.

4. $c_{1}: A \rightarrow F$ is F-linear.

5. If $B$ is a subalgebra of $A$ and $b$ is in $B$, then ch. poly ${ }_{b, B}, F$ divides ch. poly ${ }_{b, A}, F$ in $F[x]$.

We will observe in 2.2 below that our notion of characteristic polynomial on $M_{n}(F)$ is the same as the usual one. Then Prop. 1.9 contains many results that one typically proves in a linear algebra course. For example, (2) gives that $\operatorname{det}\left(a^{t}\right)=\operatorname{det}(a)$ for $a \in M_{n}(F)$ and that similar matrices have the same characteristic polynomial.

Proof. (1): Write $a=\sum_{i} \alpha_{i} a_{i}$. Then ch. poly $\cdot_{a}(a)$ is the image of min. poly ${ }_{\gamma / K}(\gamma) \in$ $A \otimes R$ under the specialization map $A \otimes R \rightarrow A \otimes F=F$ given by $t_{i} \mapsto \alpha_{i}$. Since min. poly $\cdot \gamma / K(\gamma)=0$ in $A \otimes R$, we have ch. poly $\cdot_{a}(a)=0$ in $A$.

\footnotetext{
${ }^{3}$ This paragraph may be replaced by the sentence: Field extensions are "faithfully flat". See AM69 pp. 45, 46] for a definition.
} 
(2): Suppose first that $f$ is an automorphism of $A$. The diagram

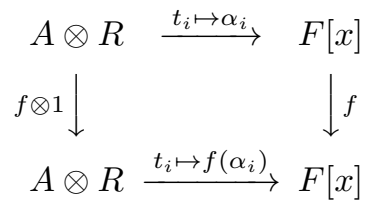

commutes. If we begin with min. poly $\cdot_{\gamma / K}$ in the upper left, we obtain ch. poly $\cdot_{a}$ in the upper right $F[x]$, and then $f\left(\right.$ ch. poly $\left._{\cdot a}\right)$ in the lower right $F[x]$. On the other hand, we obtain min. poly $\cdot f(\gamma) / K$ in the lower left and then ch. poly ${ }_{f(a)}$ in the lower right. That is, we have the desired equality ch. poly $_{\cdot f(a)}=f\left(\right.$ ch. poly $\left._{\cdot}\right)$.

The same argument works in the case where $f$ is a ring anti-automorphism, except that in the diagram we must replace $A \otimes R$ in the lower left corner with $A^{\text {op }} \otimes R$.

(3) and (4): Suppose first that $\alpha$ is not 0 . If we write the minimal polynomial of $\gamma / K$ as $\sum_{i=0}^{n} c_{i} x^{i}$ for $c_{i} \in R$, then the minimal polynomial of $\alpha \gamma / K$ is $\sum_{i=0}^{n} c_{i} \alpha^{n-i} x^{i}$. Thus

$$
\alpha^{n} \text { min. poly }{ }_{\gamma / K}(x)=\text { min. poly }{ }_{\alpha \gamma / K}(\alpha x) \text { in } R[x],
$$

and

$$
\alpha^{n} \text { ch. poly }{ }_{a}(x)=\text { ch. poly }{ }_{\alpha a}(\alpha x) \text { in } F[x] .
$$

Then we have $\alpha^{n} c_{i}(a)=\alpha^{n-i} c_{i}(\alpha a)$ for $0 \leq i \leq n$, which proves (3) for $\alpha \neq 0$. Since $c_{i}: A \rightarrow F$ is given by a polynomial in the coordinates of $a \in A$ with respect to some basis $a_{1}, \ldots, a_{m}$, this polynomial is homogeneous of degree $i$. This gives (4), as well as (3) for $\alpha=0$.

(5): Fix a basis $b_{1}, \ldots, b_{r}$ of $B$ and extend it to a basis $a_{1}, \ldots, a_{m}$ of $A$ with $a_{i}=b_{i}$ for $1 \leq i \leq r$. By analogy, set $S=F\left[t_{1}, \ldots, t_{r}\right]$, let $L$ denote the quotient field of $S$, and let $\varepsilon$ be the generic element $\sum_{i=1}^{r} t_{i} b_{i}$ in $A \otimes S$.

Write $b=\sum_{i} \beta_{i} b_{i}$ for $\beta_{i} \in F$. Let $\phi: R \rightarrow S$ be given by sending $t_{j} \mapsto 0$ for $r<j \leq m$. The image of min. poly ${ }_{\gamma / K}$ under the composition

$$
R[x] \stackrel{\phi}{\rightarrow} S[x] \stackrel{t_{i} \mapsto \beta_{i}}{\longrightarrow} F[x]
$$

is ch. poly ${ }_{b, A / F}$. Similarly, the image of min. poly ${ }_{\varepsilon / L} \in S[x]$ is ch. poly ${ }_{\cdot b, B / F} \in$ $F[x]$. The result comes from comparing $f=\phi\left(\right.$ min. poly $\left.\cdot_{\gamma / K}\right) \in S[x]$ with min. poly $\cdot_{\varepsilon / L}$. Since $\operatorname{Id}_{A} \otimes \phi: A \otimes R \rightarrow A \otimes S$ sends $\gamma \mapsto \varepsilon$, we have $f(\varepsilon)=0$, hence min. poly ${ }_{\varepsilon / L}$ divides $f$ in $L[x]$. By Gauss' Lemma, min. poly ${ }_{\varepsilon / L}$ divides $f$ in $S[x]$. Clearly, the same statement holds for their images ch. poly ${ }_{b, B / F}$ and ch. poly ${ }_{b, A / F}$ in $F[x]$, which is the desired result.

Remark 1.10. All results so far hold equally well if we allow $F$ to be a noetherian UFD and require that the $F$-algebra $A$ is a free $F$-module of finite rank. (These hypotheses are used in the proof of Lem. 1.2.)

In 1.7, $E$ must be a noetherian UFD containing $F$ for the notion of characteristic polynomial to make sense. (Since $A$ is a free $F$-module, $A \otimes E$ is a free $E$-module.) Let $L$ be the tensor product of $K$ and the quotient field of $E$, so $L$ is a field which is purely transcendental over the quotient field of $E$. The minimal polynomial for $\gamma$ over $K$ is also the minimal polynomial for $\gamma$ over $L$ by 1.7, hence characteristic polynomials are also unchanged by scalar extension in this more general setting. 
In $1.9 .5, B$ must be a free direct summand of $A$ as an $F$-module.

\section{MATRICES}

In this section, we observe that the characteristic polynomial as defined above agrees with the usual linear algebra notion of characteristic polynomial in the case where $A=M_{n}(F)$.

Lemma 2.1. The characteristic polynomial (in our sense) of a matrix in $M_{n}(F)$ has degree $\leq n$.

Proof. One can prove in an entirely elementary manner that the minimal polynomial of an $n$-by- $n$ matrix with entries in a field has degree $\leq n$, cf. Bur73, Thm. 1] or Axl95, Thm. 4.1(b)]. This applies in particular to $\gamma \in M_{n}(K)$. Specialization gives the desired conclusion.

Fix an algebraic closure $\bar{F}$ of $F$. For $a \in M_{n}(F)$, we call $\lambda \in \bar{F}$ an eigenvalue of $a$ if the kernel of $\left(\lambda I_{n}-a\right)$ is nonzero. Let $U_{\lambda}$ denote the corresponding generalized eigenspace, i.e., the set of vectors $v \in \bar{F}^{n}$ lying in the kernel of $\left(\lambda I_{n}-a\right)^{r}$ for some natural number $r$. The multiplicity $m(\lambda)$ of an eigenvalue $\lambda$ is the smallest natural number such that $\left(\lambda I_{n}-a\right)^{m(\lambda)}$ restricts to be 0 on $U_{\lambda}$.

Theorem 2.2. For $a \in M_{n}(F)$, the characteristic polynomial (as defined in 1.5) of $a$ is

$$
\left(x-\lambda_{1}\right)^{m\left(\lambda_{1}\right)}\left(x-\lambda_{2}\right)^{m\left(\lambda_{2}\right)} \cdots\left(x-\lambda_{k}\right)^{m\left(\lambda_{k}\right)},
$$

where $\lambda_{1}, \ldots, \lambda_{k}$ are the distinct eigenvalues of $a$.

In Ax195, Axler develops many of the typical properties of matrices (e.g., the existence of eigenvalues and the decomposition with respect to generalized eigenspaces) over an algebraically closed field without use of the determinant. For example, in $\S 5$ of that paper he defines the characteristic polynomial to be exactly the product displayed in the theorem. Logically, one could insert the contents of this paper at that point in his. Later in that same paper, Axler defines the determinant $\operatorname{det}(a)$ of $a \in M_{n}(F)$ to be the product of the eigenvalues with their multiplicities, i.e.,

$$
\operatorname{det}(a)=\lambda_{1}^{m\left(\lambda_{1}\right)} \lambda_{2}^{m\left(\lambda_{2}\right)} \cdots \lambda_{k}^{m\left(\lambda_{k}\right)},
$$

and he proves that this definition is equivalent to the usual alternating sum formula. For the characteristic polynomial written as in (1.8), the product (2.3) is just $c_{n}(a)$ by Thm. 2.2.

That is, our general method of associating a characteristic polynomial to an $F$ algebra gives in the case of matrices the usual ad hoc characteristic polynomial. And from first principles one can extract the formula for the determinant of a matrix in terms of its entries from the general characteristic polynomial.

Proof of Theorem 2.9. Let $B$ be the subalgebra of $M_{n}(F)$ consisting of upper triangular matrices. By 1.9.5, the characteristic polynomial of such a matrix computed as an element of $B / F$ divides the characteristic polynomial computed as an element of $A / F$. By 1.4 and 2.1, these two monic polynomials have the same degree, hence they are the same. So the characteristic polynomial ch. poly $\cdot_{a^{\prime}, A / F}$ of an upper triangular matrix $a^{\prime}$ is given by the formula in Example 1.4. 
Since the characteristic polynomial of the given matrix $a$ is unchanged under scalar extension, we are reduced to proving the theorem in the case where $F$ is algebraically closed, i.e., where $F=\bar{F}$. Here we need one somewhat sophisticated result from linear algebra: Since $F$ is algebraically closed, $a$ is similar to an upper triangular matrix $a^{\prime}$ Axl95, Thm. 6.2]. But the theorem holds for $a^{\prime}$ by the preceding paragraph and Example 1.4. Since similarity changes neither the characteristic polynomial (1.9.2) nor the eigenvalues, the theorem holds for $a$.

Matrices over rings. Apparently, we have given a canonical construction of an analogue of the characteristic polynomial and hence the determinant for any algebra over a field $F$. The reader may object that typically the characteristic polynomial and determinant are defined for $M_{n}(R)$ where $R$ is any commutative ring with 1 . But as pointed out in Remark 1.10, our method gives a characteristic polynomial and determinant for $M_{n}(\mathbb{Z})$. Since the characteristic polynomial is unchanged when we extend scalars to $\mathbb{Q}$, one sees that the general theory produces the usual characteristic polynomial for $M_{n}(\mathbb{Z})$ as well. By scalar extension we obtain characteristic polynomials for $M_{n}(R)$ where $R$ is any commutative ring with 1 , and (with a little more work) the resulting characteristic polynomial and determinant are seen to have the usual felicitous properties.

\section{Other ALGEBRAS}

Since we have produced a characteristic polynomial for an arbitrary $F$-algebra $A$, we should discuss what this polynomial is for algebras other than $n$-by- $n$ matrices.

For example, a typical topic for a first year graduate algebra course is Wedderburn's description of simple artinian rings: they are isomorphic to $M_{r}(D)$ for $D$ a skew field. Write $F$ for the center of $D$ (which is necessarily a field), and suppose that $D$ is finite-dimensional over $F$. Write $\bar{F}$ for an algebraic closure of $F$. It is standard (see e.g. Dra83, p. 31]) that $M_{r}(D) \otimes \bar{F}$ and $M_{n}(\bar{F})$ are isomorphic as $\bar{F}$-algebras for some $n$. Since characteristic polynomials are invariant under scalar extension (1.7), every element of $M_{r}(D)$ has characteristic polynomial of degree $n$. The coefficients $c_{1}$ and $c_{n}$ as in (1.8) are usually called the reduced trace and reduced norm respectively. These are very useful tools in the study of simple finite-dimensional algebras; they are especially desirable because the isomorphism $M_{r}(D) \otimes \bar{F} \cong M_{n}(\bar{F})$ identifies them with the trace and determinant that we know and love. The reduced trace and norm are typically constructed by Galois descent as in Dra83, p. 145] or [Pie82, but here we get them as a consequence of the existence of the characteristic polynomial.

In general, every $F$-algebra $A$ has its left regular representation, which is the map $A \rightarrow \operatorname{End}_{F \text {-v.s. }}(A)$ defined by sending $a \in A$ to left multiplication $L_{a}$ by $a$. This is an injective $F$-algebra homomorphism, so $a$ and $L_{a}$ have the same minimal polynomials over $F$.

Example 3.1. Let $A$ be $M_{n}(F)$ or more generally a simple ring with center $F$ such that $\operatorname{dim}_{F} A=n^{2}$. One can show that

$$
\text { ch. poly } \cdot_{L_{a}, \operatorname{End}_{F-\text { v.s. }}(A) / F}=(\text { ch. poly } \cdot a, A / F)^{n}
$$

for every $a \in A$, see e.g. Bou48, Ch. VIII, §12.3]. 
Proposition 3.3. Let $a$ be an element in a finite-dimensional F-algebra $A$. The minimal polynomial min. poly $\cdot_{a / F}$ and the characteristic polynomial ch. poly $\cdot_{a, A / F}$ have the same irreducible factors.

Proof. Fix an algebraic closure $\bar{F}$ of $F$. Different irreducible polynomials in $F[x]$ have disjoint sets of roots in $F[x]$, so it is enough to show that the minimal and characteristic polynomials have the same roots in $\bar{F}$. That is, we may assume that $F$ is algebraically closed.

Suppose first that $A$ is the algebra of $n$-by- $n$ matrices $M_{n}(F)$. This case may be argued directly at in 1.4 and 2.2. Alternately, the roots of the minimal polynomial of $a$ are the eigenvalues of $a$ [Axl97, Thm. 8.3.6], so in this case we are done by Thm. 2.2.

In the general case, the left regular representation identifies $A$ with a subalgebra of $\operatorname{End}_{F \text {-v.s. }}(A)$. Thus the polynomial ch. poly ${ }_{a, A / F} \operatorname{divides~ch.~poly~}{ }_{L_{a}}, \operatorname{End}_{F \text {-v.s. }}(A) / F$ by 1.9.5. Since $a$ and $L_{a}$ have the same minimal polynomial, it is enough to show that the minimal and characteristic polynomials of $L_{a}$ have the same irreducible factors. That is, we have reduced the proof to the case where the algebra is $M_{n}(F)$, where we already know the proposition to be true.

This proposition - and the perspective provided by its proof - allows one to drag back many nice properties of the characteristic polynomial of a matrix. For example, for our arbitrary $a \in A$ above, it is now easy to prove that $c_{i}\left(1_{A}\right)=\left(\begin{array}{c}n \\ i\end{array}\right)$; $a$ is a unit in $A$ if and only if $c_{n}(a) \neq 0$; and $a$ is nilpotent in $A$ if and only if it has characteristic polynomial $x^{n}$. With more complicated arguments, one can also prove that ch. poly. ${ }_{a}(x)=c_{n}\left(x 1_{A}-a\right)$ Jac68, p. 225], which is analogous to the typical definition of the characteristic polynomial; and $c_{n}\left(a a^{\prime}\right)=c_{n}(a) c_{n}\left(a^{\prime}\right)$ for $a, a^{\prime}$ in $A$ [Jac68, p. 235], which is a generalization of the equation $\operatorname{det}\left(a a^{\prime}\right)=\operatorname{det}(a) \operatorname{det}\left(a^{\prime}\right)$.

Remark 3.4. An early reader of this paper preferred a different viewpoint. He was not troubled by the definition of determinant and characteristic polynomial for matrices. For $A$ a simple ring that is $n^{2}$-dimensional over its center $F$, he defined the characteristic polynomial of an element $a \in A$ as the $n$-th root of the characteristic polynomial of $L_{a}$.

Unfortunately, one cannot use this method to recover our characteristic polynomial of an arbitrary finite-dimensional $F$-algebra $A$ because the relation (3.2) need not hold. Also, there is no mathematical reason to prefer the left over the right regular representation (defined in the obvious manner as $a \mapsto R_{a}$ ), and the characteristic polynomials of $L_{a}$ and $R_{a}$ may differ. Adrian Wadsworth pointed out that the upper triangular matrices from Example 1.4 provide an example of both of these difficulties. In particular, the generic element $\gamma$ has

$$
\text { ch. poly } \cdot_{a}(\gamma)=\left(x-t_{1}\right)^{n}\left(x-t_{2}\right)^{n-1} \cdots\left(x-t_{n}\right)^{2}\left(x-t_{n}\right)
$$

and

$$
\text { ch. poly } R_{a}(\gamma)=\left(x-t_{1}\right)\left(x-t_{2}\right)^{2} \cdots\left(x-t_{n}\right)^{n-1}\left(x-t_{n}\right)^{n} \text {. }
$$

\section{REFERENCES}

[Alb61] A.A. Albert, Structure of algebras, AMS Coll. Pub., vol. 24, AMS, Providence, RI, 1961, revised printing.

[AM69] M.F. Atiyah and I.G. MacDonald, Introduction to commutative algebra, Addison-Wesley, Reading, MA, 1969.

[Ax195] S. Axler, Down with determinants!, Amer. Math. Monthly 102 (1995), no. 2, 139-154. 
[Ax197] S. Axler, Linear algebra done right, second ed., Undergraduate Texts in Mathematics, Springer, 1997.

[Bou48] N. Bourbaki, Algèbre, Hermann, Paris, 1948.

[Bre01] O. Bretscher, Linear algebra with applications, second ed., Prentice Hall, 2001.

[Bur73] M.D. Burrow, The minimal polynomial of a linear transformation, Amer. Math. Monthly 80 (1973), 1129-1131.

[Deu48] M. Deuring, Algebren, Chelsea, 1948.

[Dic38] L.E. Dickson, Algebras and their arithmetics, G.E. Stechert, New York, 1938.

[Dra83] P.K. Draxl, Skew fields, London Math. Soc. Lecture Note Series, vol. 81, Cambridge University Press, Cambridge-New York, 1983.

[Han96] J. Hannah, A geometric approach to determinants, Amer. Math. Monthly 103 (1996), no. 5, 401-409.

[Her75] I.N. Herstein, Topics in algebra, second ed., Xerox College Publishing, Lexington, Mass., 1975.

[Hun80] T. Hungerford, Algebra, second ed., Springer, 1980.

[Jac41] C.G.J. Jacobi, De formatione et proprietatibus determinantium, J. Reine Angew. Math. 22 (1841), 285-318, (= Ges. Werke, vol. III, pp. 355-392).

[Jac43] N. Jacobson, The theory of rings, Mathematical Surveys, vol. 2, American Mathematical Society, New York, 1943.

[Jac63] N. Jacobson, Generic norm of an algebra, Osaka Math. J. 15 (1963), 23-50, (= Coll. Math. Papers, vol. 2, pp. 489-515).

[Jac68] N. Jacobson, Structure and representations of Jordan algebras, AMS Coll. Pub., vol. 39, AMS, Providence, RI, 1968.

[Jac75] N. Jacobson, PI-algebras: an introduction, Lecture Notes in Mathematics, vol. 441, Springer-Verlag, Berlin, 1975.

[Kno94] E. Knobloch, From Gauß to Weierstraß: determinant theory and its historical evaluation, The intersection of history and mathematics, Science Networks, vol. 15, Birkhäuser, 1994, pp. 51-66.

[Lax97] P.D. Lax, Linear algebra, John Wiley \& Sons Inc., New York, 1997.

[Pie82] R.S. Pierce, Associative algebras, Graduate Texts in Mathematics, vol. 88, Springer, 1982.

[Sch91] G. Scheffers, Zurückführung complexer Zahlensysteme auf typische Formen, Math. Annalen 39 (1891), 293-390.

[Stu89] E. Study, Über Systeme von complexen Zahlen, Nachrichten von der Königlichen Gesellschaft der Wissenschaften zu Göttingen (1889), no. 9, 237-268.

[Web61] H. Weber, Lehrbuch der Algebra, third ed., vol. 1, Chelsea, 1961?

[Wei] K. Weierstrass, Zur Determinantentheorie, note based on lectures given in 1886/87. Appeared posthumously in Mathematische Werke, (J. Knoblauch, ed.), vol. III, 1903, pp. 271-286.

UClA Dept. of Mathematics, Los Angeles, CA 90095-1555, USA

E-mail address: skip@member.ams.org 